\title{
Architecture of the data aggregation and streaming system for the European Spallation Source neutron instrument suite
}

\author{
A.H.C. Mukai, ${ }^{a, 1}$ M.J. Clarke, ${ }^{a}$ M.J. Christensen, ${ }^{a}$ J.M.C. Nilsson, ${ }^{a}$ M.G. Shetty, ${ }^{a}$ \\ M. Brambilla, ${ }^{b}$ D. Werder, ${ }^{b}$ M. Könnecke, ${ }^{b}$ J. Harper, ${ }^{c}$ M.D. Jones, ${ }^{d}$ F.A. Akeroyd, ${ }^{c}$ C. Reis, ${ }^{e}$ \\ G. Kourousias ${ }^{e}$ and T.S. Richter ${ }^{a}$ \\ ${ }^{a}$ European Spallation Source ERIC, \\ Ole Maalфes Vej 3, 2200 Copenhagen N, Denmark \\ ${ }^{b}$ Paul Scherrer Institut, \\ 5232 Villigen PSI, Switzerland \\ ${ }^{c}$ ISIS Neutron and Muon Source, Science and Technology Facilities Council, \\ Rutherford Appleton Laboratory, Didcot, OX11 0QX, United Kingdom \\ ${ }^{d}$ Tessella, \\ 26 The Quadrant, Abingdon Science Park, Abingdon, Oxfordshire, OX14 3YS, United Kingdom \\ ${ }^{e}$ Elettra Sincrotrone Trieste, \\ Strada Statale 14, km 163.5, 34149 Basovizza, Trieste, Italy \\ E-mail: afonso.mukai@esss.se
}

AвSTRACт: The European Spallation Source (ESS) will provide long neutron pulses for experiments on a suite of different instruments. Most of these will perform neutron data acquisition in event mode, i.e. each detected neutron will be characterised by one absolute timestamp and pixel identifier pair. Slow controls metadata from EPICS, such as sample environment measurements and motor positions, will also be timestamped at their source, so that all data and metadata are streamed as a list of events instead of histograms. A flexible data aggregation and streaming system is being developed combining both open source third-party software and in-house development. This is to be used at ESS and other neutron scattering facilities like ISIS and SINQ, replacing legacy solutions by a shared software collection maintained by a cross-facility effort. The architecture of the Apache Kafka-based system, its metadata forwarding and NeXus file writing components are presented, along with test results demonstrating their integration and the scalability in terms of performance.

Keywords: Software architectures (event data models, frameworks and databases); Computing (architecture, farms, GRID for recording, storage, archiving, and distribution of data); Data acquisition concepts

ARXIV EPRINT: 1807.10388

${ }^{1}$ Corresponding author. 


\section{Contents}

1 Introduction 1

2 Event mode acquisition $\quad 2$

2.1 ESS instrument data rates 4

2.2 Timing system and timestamping of data 4

3 Data aggregation and streaming $\quad 5$

3.1 Apache Kafka 6

3.2 Serialisation and schemas 7

3.3 EPICS Forwarder 8

3.4 Writing data to file 8

4 Tests and results $\quad 10$

$\begin{array}{lll}4.1 & \text { Integration tests } & 10\end{array}$

4.2 Performance tests 11

$\begin{array}{lll}4.3 & \text { Deployments at operating facilities } & 13\end{array}$

5 Conclusion and future work $\quad 13$

\section{Introduction}

The European Spallation Source (ESS) is a spallation neutron source under construction in Lund, Sweden. A linear accelerator produces a high intensity proton beam which is guided on a tungsten target, with the spallation process generating fast neutrons from the heavy tungsten atoms. These fast neutrons are slowed down in a moderator and then guided onto samples with which they will interact. From the interaction of samples with neutrons, scientists can learn about the properties of materials. Applications include fundamental solid state physics, materials science, crystallography, biology and archaeology.

The ESS will operate as a user facility: external users will come to perform neutron scattering experiments using the provided high brightness, long pulse neutron beam. A suite of instruments covering different techniques, such as imaging, small angle scattering, reflectometry, diffraction and spectroscopy, will be able to use the unprecedented flux there for experiments [1].

Due to the high brilliance neutron beam, high performance computing is needed for data processing. Located in Copenhagen, Denmark, the ESS Data Management and Software Centre (DMSC) develops, maintains and operates a software and hardware infrastructure for tasks ranging from data acquisition, aggregation and streaming to reduction and analysis, both in real time and offline. The DMSC works in close collaboration with in-kind and project partners across Europe, 
including Paul Scherrer Institut (PSI) in Switzerland, the Science and Technology Facilities Council (STFC) in the United Kingdom, and Elettra in Italy.

Most ESS instruments will acquire neutron data from detectors in event mode, i.e. as a list of pixel number-timestamp pairs. Slow metadata obtained from devices using the Experimental Physics and Industrial Control System (EPICS) also come in the form of timestamped values [2]. An aggregation and streaming software architecture based on Apache Kafka [3] will make these data available to applications performing tasks such as file writing using the NeXus format [4] and live data reduction with feedback to the experiment control as well as visualisation.

Due to the high brightness of the ESS neutron beam, the solutions for data acquisition must be able to cope with correspondingly high data rates, making performance an important requirement, as will be discussed in this article. Considering the future possibilities of expansion of the ESS instrument suite and of upgrades to initial instruments, good scalability of the solution is essential. The selected open source components and the design of the in-house developed software take this into consideration.

In this article, the overall architecture of the data aggregation and streaming system is described, with a focus on the core components: Apache Kafka, the EPICS to Kafka Forwarder and the NeXus File Writer. Section 2 discusses data acquisition in event mode, the requirements arising from it and also the ESS approach to timing and timestamping. In section 3, the architecture and components are introduced and discussed. The current deployments of the system as well as integration and performance scalability tests are presented in section 4 , with conclusions summarised in section 5 .

\section{Event mode acquisition}

Acquisition of neutron instrument data is frequently done in histogram mode, in which detector data over a region is acquired for an interval and presented as a set of accumulated counts. In event mode acquisition, each neutron count on the detector generates an individual event consisting of a pixel number, which uniquely identifies the location where the neutron hit the detector, and a timestamp. Acquired data are sent forward as a sequence of events. Figure 1 illustrates the difference between these two modes of data acquisition.

Postponing histogram generation provides additional flexibility to the data acquisition process, as the conversion of neutron detection events into a histogram results in loss of information (the individual timestamps are lost and the final histogram only gives information as detailed as the binning and integration time allow). Not only can the data in an event list later be binned in any desired resolution, individual (sets of) events can also be filtered out depending on metadata parameters like, for example, sample environment information. In the ESS, event mode acquisition will occur with no hardware veto, that is, data acquisition will not be automatically inhibited should a neutron chopper be out of phase. As chopper metadata will be recorded, filtering can be done by discarding events for intervals where choppers were out of phase, or by taking into account the instantaneous chopper phases, as well as source and target parameters and correcting the neutron wavelengths for it.

The data aggregation and streaming system will receive neutron data and metadata from different sources. Event data come from Event Formation Unit (EFU) software running on servers that interface with the detector readout electronics and obtain the pixel identifier-timestamp pair from 

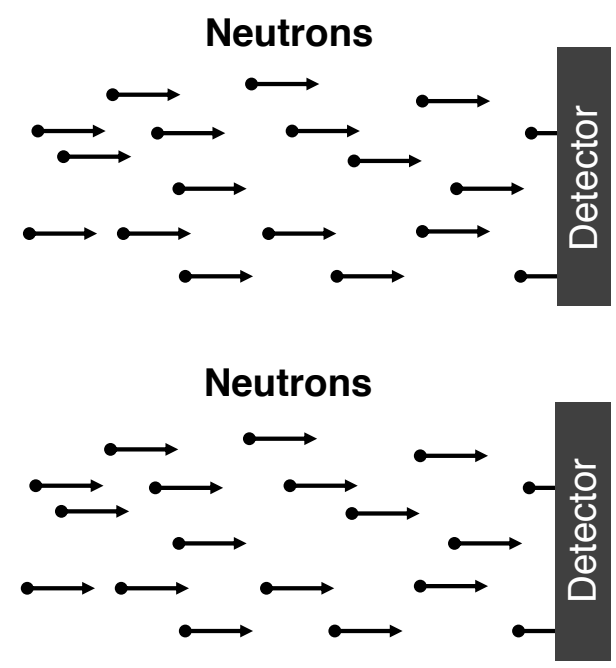

Histograms

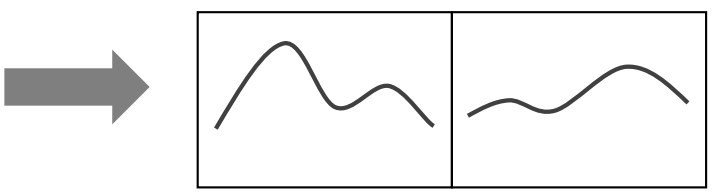

Events

Pixel ID $\quad 42 \quad 31 \quad 14 \quad 00 \quad 99$

Figure 1. Data acquisition in histograms and event mode. In the first mode, counts from neutrons incident on the detector are accumulated and sent as a histogram. In the latter, pairs of timestamp and pixel identifier are sent for each detected neutron in a list of individual events.

the raw detector data [5]. Figure 2 shows a screenshot of the detector commissioning tool Daquiri [6] with examples of event data before and after processing by the EFU. EPICS metadata will be sent by the EPICS to Kafka Forwarder application, which subscribes to value updates from multiple Input/Output Controllers (IOCs) that publish the readings for beamline devices like temperature controllers, motors and choppers. The highest data rates are expected to arise from neutron event data; these will be discussed next, followed by the ESS approach to timing and timestamping.

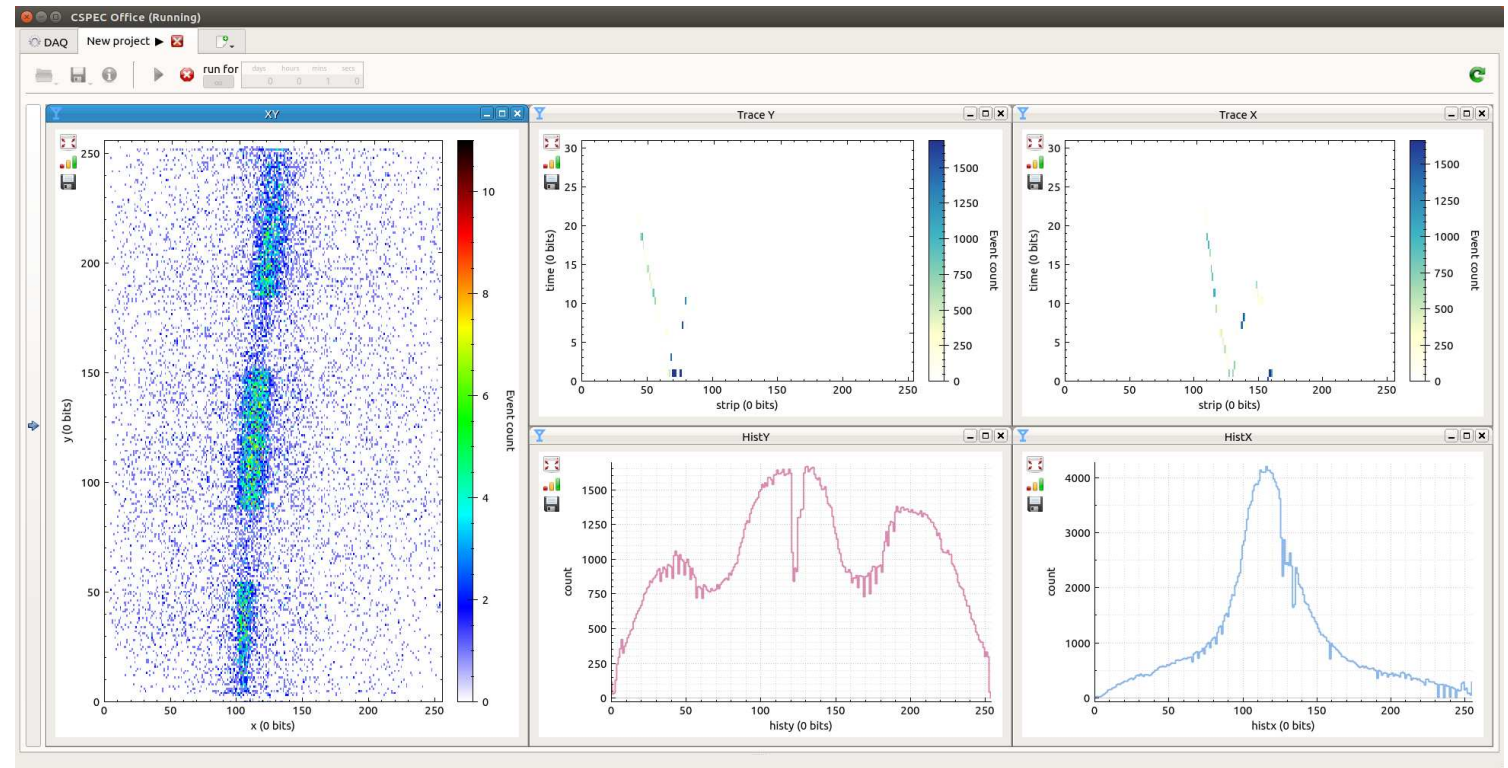

Figure 2. Screenshot of the detector commissioning tool Daquiri with detector prototype test data. The two smaller plots on the top show event traces (for a single event) before processing by the EFU. The plot on the left shows event counts on a detector surface after processing by the EFU. 


\subsection{ESS instrument data rates}

As a result of the high neutron beam brightness, the expected event data rates for ESS instruments are correspondingly high. Each detector pixel identifier and timestamp will be encoded in a pair of 32-bit numbers [7]; using these values, table 1 presents estimates of the data rates expected to be sent from the EFUs into the aggregation and streaming system [5].

Table 1. Anticipated neutron and detector event rate estimates for some early ESS instruments.

\begin{tabular}{|lcc|}
\hline Instrument & $\begin{array}{c}\text { Global Average Rate } \\
{[\mathrm{MHz}]}\end{array}$ & $\begin{array}{c}\text { Data Rate } \\
{[\mathrm{MB} / \mathrm{s}]}\end{array}$ \\
\hline C-SPEC & 10 & 80 \\
ESTIA & 500 & 4000 \\
FREIA & 100 & 800 \\
LoKI & 37 & 300 \\
NMX & 5 & 40 \\
SKADI & 37 & 300 \\
T-REX & 10 & 80 \\
\hline
\end{tabular}

As the table shows, expected rates cover a range of almost three orders of magnitude, from $10 \mathrm{MB} / \mathrm{s}$ to $4 \mathrm{~GB} / \mathrm{s}$. In addition to the bare detector neutron rate, the metadata from beam monitors, choppers, (fast) sample environments and so on also need to be considered. It is expected that the total metadata volume can be up to the order of the neutron data for an average instrument. This sets the requirements for the rates the system must support for online visualisation and file writing. The accelerator will be ramped up in power over a few years and instruments will be built and commissioned over a period of time, so the ESS will not initially operate at maximum rates; the aggregation and streaming solution should therefore be scalable, allowing it to expand by adding more resources as necessary.

\subsection{Timing system and timestamping of data}

For devices that produce data or metadata, all readout values will be timestamped at the source (with deterministic latency) using information from the ESS timing system. In this system a tree topology of event receivers with an event generator at the root is used for distributing trigger and clock signals, timestamps, and accelerator parameters across the facility with deterministic and compensated latency. In normal operations, the ESS neutron pulses of $2.86 \mathrm{~ms}$ duration will have a repetition rate of $14 \mathrm{~Hz}$. The timing system will make available beam parameters such as the pulse length and proton beam energy and will be integrated with EPICS [8].

Neutron detector data acquisition in event mode relies on information that is provided by the ESS timing system, such as accurate timestamps for neutron events coming from the detector readout electronics and accelerator pulse sequence numbers. Events are accumulated and sent in a serialised message (see section 3.2) that includes a 64-bit pulse time in nanoseconds since an epoch and an array of 32-bit neutron timestamps in nanoseconds from pulse time. Furthermore, to be able to make sense of data and process the events, sample conditions must be known at any given instant; for that, the timestamped metadata are used, using values provided by EPICS process 
variables. As all the timestamping happens at the data source, later transport over normal computer networks, software data aggregation and processing in standard non-realtime systems and so on do not distort the data by adding latency. Latency times for the data must be kept reasonably low to allow live experiment data visualisation by the user and enable useful automatic feedback from live data processing to the control of the ongoing experiment.

\section{Data aggregation and streaming}

Data acquisition in event mode produces a stream of pixel identifier-timestamp pairs, which are subsequently processed by other applications and combined with metadata to enable a user to make sense of the experiment being performed. By selecting a system architecture based on aggregation and streaming [9], the data publishers do not have to be directly connected to every data subscriber, thus decoupling the two categories and allowing them to scale separately. A common serialisation format is needed to ensure the different applications all understand the data in the same way.

An overall view of the adopted system architecture is presented in figure 3. The data sources depicted in that diagram are the Event Formation Units; the Fast Sample Environment, which sends event data from fast readout systems such as alternating electric and magnetic fields; and the EPICS to Kafka Forwarder, which sends experiment metadata from EPICS IOCs. Subscribing to these data are the File Writer, which generates files conforming to the NeXus format [4] for offline reduction and analysis, and the data reduction application Mantid [10] performing live data reduction to enable online visualisation. The streams of data are brokered through an Apache Kafka cluster, which is also used for sending and receiving control and status messages. In the next sections the central components for aggregation and streaming are discussed in more detail.

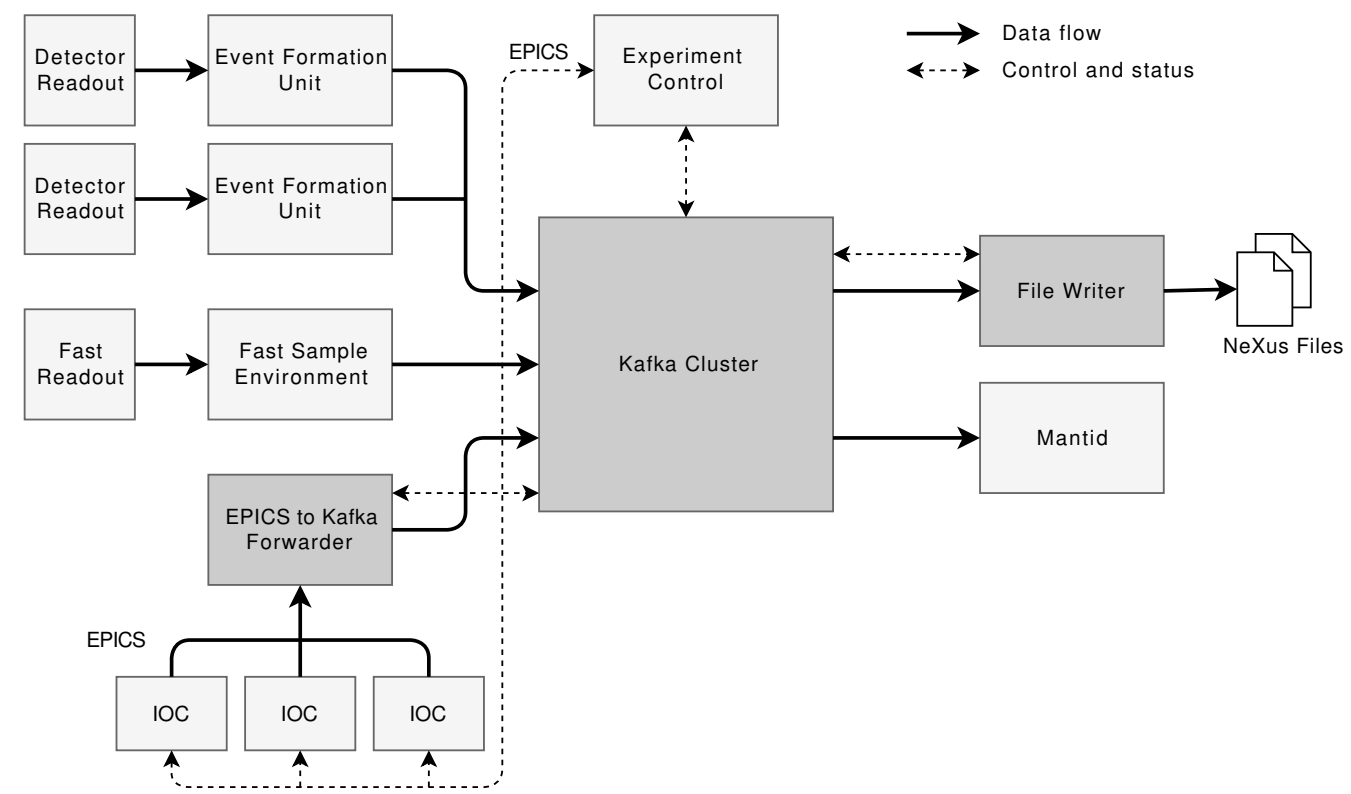

Figure 3. System architecture overview. Apache Kafka provides the central infrastructure that connects data producers and consumers. Components in dark grey are the main focus of the data aggregation and streaming and are described in this publication. 
Instead of using specifically-tailored solutions for the ESS, the data aggregation and streaming system relies on a number of open source tools and libraries, and the applications developed in house are also generic and configurable. While specific solutions can be optimised to minimise resource usage considering detailed information about the systems they cater to, using more generic tools provides leverage through a larger developer and user community, alleviating the local development and maintenance work that can focus on domain-specific applications. The decoupling of the system components combined with the common serialisation format allows different facilities to plug in their own specific applications, while using the other parts of the infrastructure. An example of such use of the system by the ISIS Neutron and Muon Source is presented in section 4.3.

\subsection{Apache Kafka}

Apache Kafka is an open source distributed streaming platform that provides a scalable solution for applications to publish and subscribe to streams of data [3]. Kafka has been selected as the central technology for aggregation and streaming based on its rich set of implemented functionality, scalability, large and active user and developer communities, and the availability of good documentation [11].

In Kafka terminology, the producers (publishers) write data to named channels known as topics, to which consumers can subscribe. A cluster is formed by a number of Kafka brokers, with the possibility of partitioning topics across different brokers for scalability and replicating them for availability in case of broker failure. Data are persisted to disk for a configurable time or storage limit in the cluster, allowing consumers to obtain historical data. The cluster rate capacity can be increased by adding more brokers and partitioning topics across them.

Figure 4 illustrates how topic partitioning can be used to allow detector readout to scale by writing parts of the event streams to different Kafka brokers. It also shows that the intention is to use one common Kafka installation for all instruments. This shared system can cope with occasional peak loads from instruments far above their average rate, without needing to over supply capacity at every instrument. In addition to the depicted scaling out, Kafka will also be used to provide redundancy by duplication of the topics for the raw data storage, so experimental data is safely persisted early on.

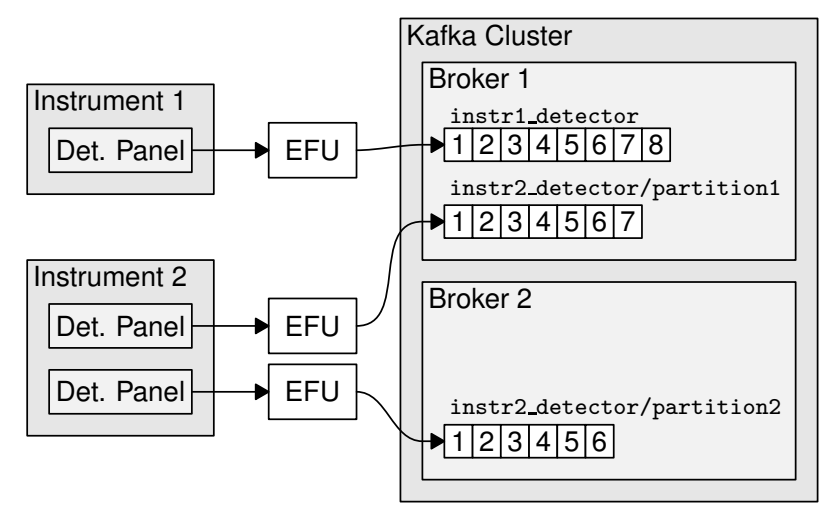

Figure 4. Neutron detector data from Event Formation Units sent to different Apache Kafka topics and partitions. Partitioning of topics across different Kafka brokers provides scalability to handle high data rates, as each broker runs on a server with dedicated network interfaces and storage resources. 


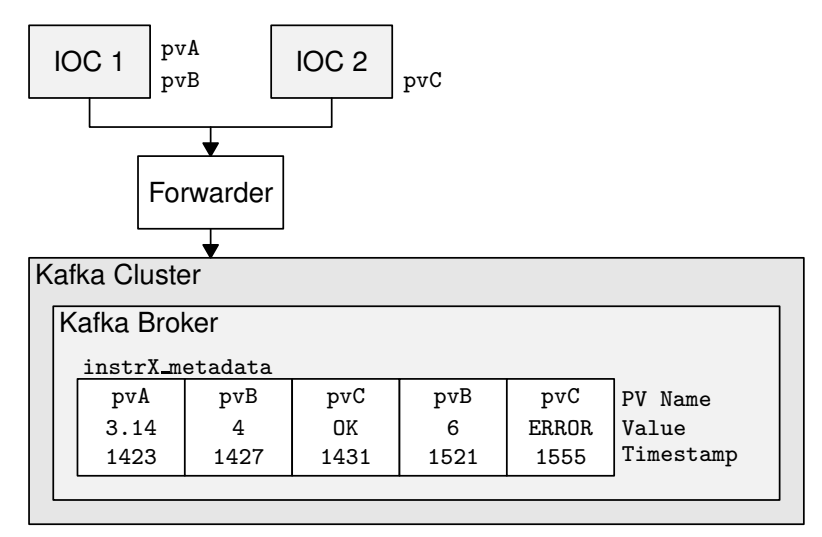

Figure 5. EPICS to Kafka Forwarder multiplexing data from different EPICS process variables (PVs) using the same Kafka topic.

Fast sample environment data will also be sent to the cluster using an EFU-based solution. For lower rate data sources, such as EPICS metadata, whose values are updated at rates in a typical range of $0.1 \mathrm{~Hz}$ to $100 \mathrm{~Hz}$, the same Kafka topic can be used for multiple sources. The EPICS to Kafka Forwarder serialises and multiplexes different sources over a single topic, as illustrated in figure 5.

\subsection{Serialisation and schemas}

As the aggregation and streaming system is comprised of distributed producers and consumers that exchange data and commands through Apache Kafka, a well-defined common set of schemas is required to ensure the applications can make sense of the data being exchanged. Google FlatBuffers, an efficient cross-platform serialisation library [12], has been chosen for serialising neutron event data and beamline metadata. A common Streaming Data Types repository with an agreed procedure for introducing changes is used for controlling the schemas that define the message format [7]. Schemas can include other schema files, a feature that is used in the neutron event schema to add facility-specific information to them.

Serialised messages are not self-describing (i.e. they cannot be deserialised without knowledge of the schema), but include a four-byte file identifier; this is used to identify the schema required to deserialise the message by the consuming application. A convention of prefixing the schema file name with the identifier has been adopted to help identify the files in the repository. The FlatBuffers library allows schema fields to be optional or required, and fields can be added and deprecated. FlatBuffers provides a compiler that takes schemas as input and generates source files in a number of programming languages, including $\mathrm{C}++$ and Python, the main languages used by the ESS aggregation and streaming programs. These source files provide language-specific methods for serialising and deserialising messages to and from buffers according to a given schema.

The schema used by the EFU for sending event data to the Kafka cluster includes a global timestamp associated with a neutron pulse and a pair of arrays containing event timestamps and pixel numbers; buffer size is dominated by the number of events in it. For message sizes of about $1 \mathrm{MB}$ (see figure 8 and the discussion in section 4.2), this means hundreds of thousands of events can be accumulated by the EFU in each message. On high data rate instruments, buffers can be filled 
with events and sent after a certain message size has been reached, while for lower rate instruments messages can be sent for each pulse, thus balancing throughput and latency concerns.

Apache Kafka is also used for the exchange of control and status messages between applications. Examples of control messages include commands for starting and stopping file writing by the Experiment Control Program, and both the EPICS to Kafka Forwarder and the NeXus File Writer send status messages reporting their state and activity periodically. These messages are currently sent in JSON format to dedicated Kafka topics, with a low rate of the order of $1 \mathrm{~Hz}$.

\subsection{EPICS Forwarder}

The EPICS to Kafka Forwarder [13] is the component in the data aggregation and streaming architecture that monitors a list of EPICS process variables and makes these updates available for the rest of the components via the Kafka cluster.

Monitoring of the process variables is done using EPICS version 4, which supports both the older Channel Access protocol and the newer pvAccess [14]. The list of monitored variables can be set in a configuration file that is read by the EPICS to Kafka Forwarder at startup and can also be modified via JSON command messages, which are delivered through Kafka.

One or more conversion modules can be attached to each monitored process variable, which take the responsibility to convert the received EPICS value into a buffer for the desired schema, as described in section 3.2. It is also possible to attach an optional conversion module to several monitored process variables to cater for more elaborate setups. The converted update is delivered to the configured topic in the Kafka cluster (see section 3.1) via the $\mathrm{C} / \mathrm{C}++$ librdkafka library [15], which handles the network connections, buffering and delivery reports.

The EPICS to Kafka Forwarder features a highly parallel design based on worker queues and a configurable number of worker threads. It is designed to handle updates at a high frequency, which is achieved by keeping the EPICS update callback as lightweight as possible, while still providing the functionality to enable concurrency in the later stages of the forwarder pipeline. The update of the process variable can go through the pipeline stages while the thread that invokes the monitor callback, which is owned by the EPICS library, has already returned control to the library.

At runtime, the forwarder sends status and statistics about the monitored process variables to the Kafka cluster in JSON format. This information is useful for system monitoring by the Experiment Control Program and for diagnostics.

\subsection{Writing data to file}

While the Kafka cluster partitions persist the full stream of experimental data in roughly temporal order as they are measured, it is beneficial for the performance of offline data reduction or analysis algorithms to be able to access the data in larger homogeneous chunks. File-based storage is also well established in computing architectures and in the scientific community with agreed, self describing formats that can be a long term scientific record for experiments at the ESS.

NeXus is a common data format for neutron, X-ray and muon science [4] and is based on the Hierarchical Data Format 5 (HDF5) [16]. Many institutions and existing pieces of software use NeXus. By opting to store data in this format the ESS benefits from existing support in software, including Mantid [10]. The NeXus File Writer [17] creates HDF5 files that contain a configured set of the available data and metadata from experiments. 
HDF5 stores data in a format analogous to a filesystem. Directory-like objects called groups contain child groups and datasets. Datasets are analogous to files and contain a scalar value or a multidimensional array of data which may also be compressed; datasets and groups can each have attributes containing supporting metadata. Links can be used in place of groups or datasets and can link to locations in the same file or in external files.

The NeXus format defines a collection of classes, each of which has a set of required or optional child datasets, classes and attributes. HDF5 groups in a NeXus file declare their conformance to a NeXus class with an attribute called NX_class, which has a value of the name of the class. Figure 6 depicts this for a simple hierarchy. Classes exist to support a wide range of different data [18], including images, histograms and time series data. They can also describe details about how the experiment was carried out, such as who carried out the experiment and when, information about the sample being studied, what components comprise the instrument, the instrument's geometry, and any other data required for the analysis of an experiment at a neutron source or synchrotron facility.

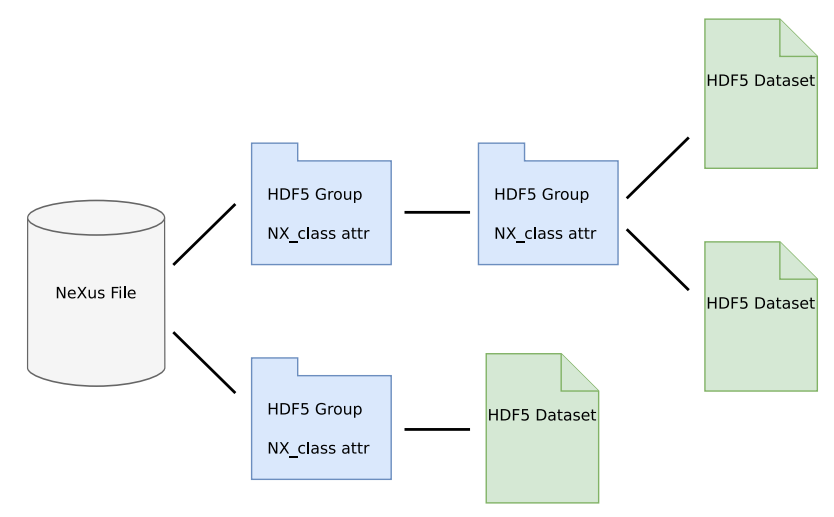

Figure 6. Diagram showing the hierarchy of a NeXus file, which comprises HDF5 datasets and groups that conform to NeXus class definitions.

As mentioned in section 2, key data types for the ESS are neutron detection events and time series data from choppers or measurements of the sample environment. These types of data are well supported in NeXus by the NXevent_data and NXlog classes, respectively. Each of these classes contain datasets of values and corresponding timestamps. These time-value datasets are supplemented by cue datasets which record the index in the value and timestamp datasets at particular times. If only a subset of the time range recorded in the value-timestamp datasets is required to be read from file, this can be achieved by reading the smaller cue datasets and using the indices into the large datasets. This is therefore possible without having to read the entire time-value datasets, which may be slow or impossible in the case that they exceed available memory. This system is also particularly well suited to streamed data, as neutron detection events are batched in messages and a cue can be recorded for each message.

The file writer writes NeXus files upon requests received in the form of JSON commands via a Kafka topic. The request contains the structure of the NeXus file which is to be written. This description of the NeXus structure can contain the HDF5 group hierarchy, attributes, datasets which are already known at the time when the command is sent, and placeholders for datasets which are to be written as the corresponding data messages appear on configured Kafka topics. Because the file 
writer has access to historic data through the Kafka cluster, the commands can contain timestamps for the time range of the data to be written, which can be in the past or in the future. If start and stop times are not defined, the writing of a file starts as soon as the command is received by the NeXus File Writer and it stops on a corresponding stop command.

The data streams, which are specified in form of the NeXus structure in the JSON command, are handled by specified HDF5 writer modules. They process the messages that arrive on the configured Kafka topic and are responsible for any additional processing and the actual writing of that data to the HDF5 file. This modular design allows easily extending the file writer to cater for new FlatBuffers schemas, for more specialised output formats or optimised ways of writing data to file.

\section{Tests and results}

To verify and demonstrate the viability of the proposed approach to data aggregation and streaming, a series of tests have been performed, covering the integration of the components and their performance. Some of the tests are automated and run as new versions of the applications are created, in a local computing infrastructure with virtual machines at the DMSC; others are run on demand on dedicated hardware. Furthermore, the system has been deployed to operating neutron facilities for evaluation. In the following sections, the different types of tests and their results are presented, along with a discussion of the deployments of the system to operating facilities.

\subsection{Integration tests}

An automated integration testing regime including the data aggregation and streaming software is in place in the software build and test infrastructure at the DMSC, consisting of a Jenkins continuous integration server using the configuration management and orchestration tool Ansible. A test is triggered every time changes are made to the code bases of the Event Formation Unit, EPICS to Kafka Forwarder and NeXus File Writer. Raw data from detector prototype measurements are streamed to the EFUs, which process the signals into events and forward them to the Kafka cluster. A simulation EPICS server is used to generate process variable data for the EPICS forwarder, and the file writer is started and configured to write detector data and EPICS metadata to a NeXus file. Figure 7 shows the current setup; results are made available through the Jenkins server web interface, while metrics and logs can be visualised with Grafana and Graylog, respectively.

The integration test verifies the correct number of events have been sent from the EFUs. Metrics and $\log$ data are stored for the test runs and provide benchmark information such as Kafka data rates. A script verifies the data written to the NeXus file.

At the moment three dedicated servers are available at an ESS integration laboratory, where other beamline equipment such as motion control and sample environment devices are also located for testing purposes, along with detector prototypes. These servers are operated for manual tests and have been used for a successful demonstration of streaming event data from EFUs into Kafka with live visualisation. Work is in progress to integrate the Experiment Control Program and Fast Sample Environment application with the devices in the laboratory, and this will allow those components to be tested with the data aggregation and streaming system. 


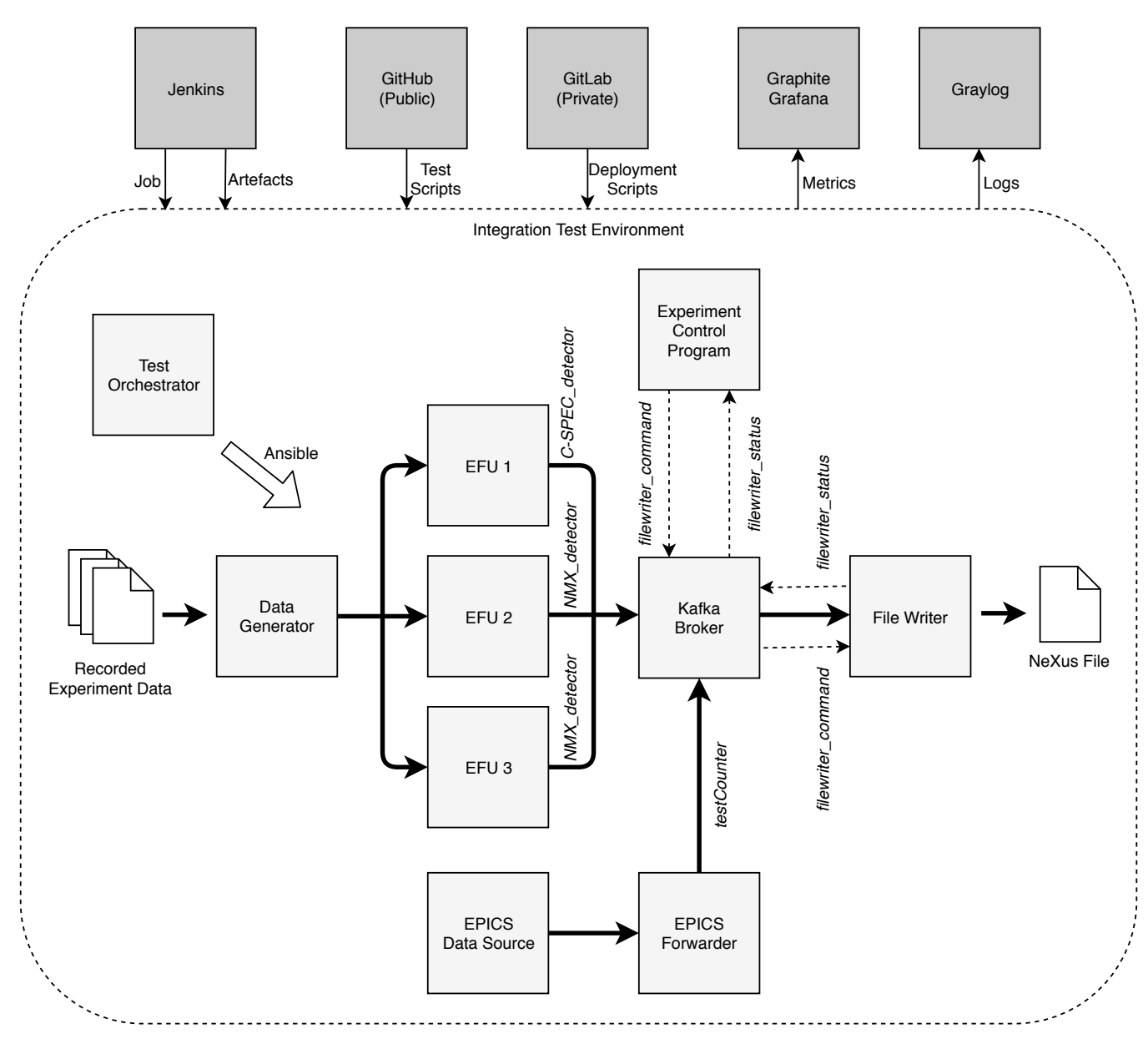

Figure 7. Integration test setup. The Jenkins build server triggers the test, which runs in virtual machines in the DMSC computing infrastructure (depicted in light grey). The test orchestrator node uses Ansible to connect to the other virtual machines and run the required services and commands. Data flow is represented by bold lines, status and commands by dashed lines, and topic names are written in italics.

\subsection{Performance tests}

The performance of the aggregation and streaming system, including the NeXus File Writer, was investigated at PSI using four Intel Xeon E5-2697 v4 $2.60 \mathrm{GHz}$ servers, equipped with $252 \mathrm{~GB}$ of RAM and a GPFS file system connected via 4x Infiniband FDR. This system is limited by the fact that all the nodes share the same I/O interface; furthermore, the same interface is used for communications to achieve optimal communication speed during the tests. On this system, a filesystem write performance test using 4 threads resulted in a $5.8 \mathrm{~GB} / \mathrm{s}$ rate, while internode network communication over Infiniband tests using 4 threads showed bandwidths above $3 \mathrm{~GB} / \mathrm{s}$ for message sizes greater than $0.1 \mathrm{MB}$.

Tests were performed using a virtual implementation of the PSI AMOR [19] instrument as data source. The output of a real measurement is converted into a neutron event stream and sent to a Kafka cluster; the number of events recorded in a single message can be varied in order to test the dependence of the system on the message size. A different number of brokers and partitions were used. 


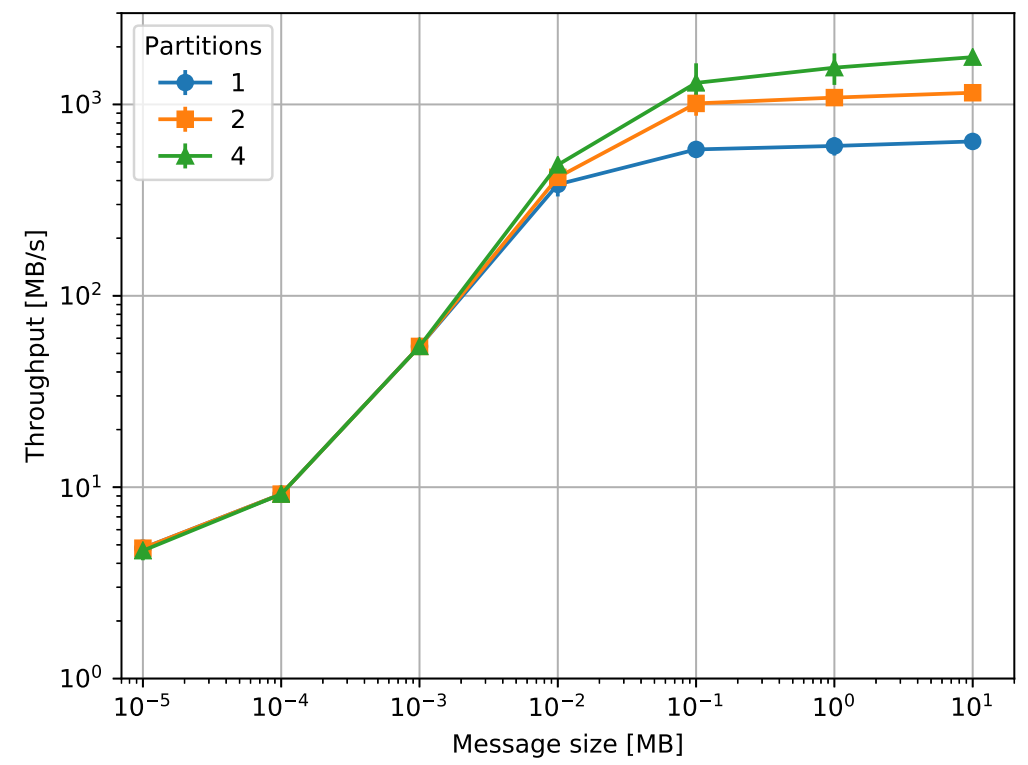

Figure 8. Kafka throughput as a function of the message size and the number of partitions. Two servers run the brokers, while a third server runs the event producer.

Figure 8 shows the producer performance achieved using four Kafka brokers as a function of the message size and the number of partitions. The figure demonstrates that the overhead of using many small messages degrades the performance; this can be acceptable for infrequent metadata updates, but the bulk of the neutron data needs to be sent in larger packets, as done by the EFUs, to reach the required throughput. This system scales well with the number of producers when using different topics and the aggregate throughput of two producers is roughly double what is shown in the figure.

Figure 9 shows the results of scalability tests with a producer, the Kafka cluster and a file writer. For a different number of servers, the total throughput was measured varying the number of brokers, using one partition per broker and a $1 \mathrm{MB}$ message size. The file writer runs on a separate server, but in the setup with three broker machines, the producer shares the server with (at least) one Kafka broker. This may have a negative effect on the performance on the three server case (green line). Otherwise the data suggests a very linear scaling of the achievable throughput with the number of deployed servers or broker processes and in turns verifies the arguments made in 3.1 for choosing Kafka: that it enables an incremental growth of the infrastructure with raising need of the scientific instruments as the ESS builds up, always keeping up with the requirements.

The performance of the HDF5 writing component is essentially limited by the available I/O. To be able to write HDF5 files at the expected high data rates, the file system layer has to be backed by an appropriately fast I/O layer. Furthermore, the File Writer exposes some parameters for fine tuning in order to achieve the best performance. On a server with Kafka messages preloaded in RAM, a single process approach sustains about $1.2 \mathrm{~GB} / \mathrm{s}$, while a parallelised implementation using Message Passing Interface (MPI) [20] can reach about $4.8 \mathrm{~GB} / \mathrm{s}$; a broad discussion on the writing performances can be found in [21], including the effects of chunking, message size and buffering. 


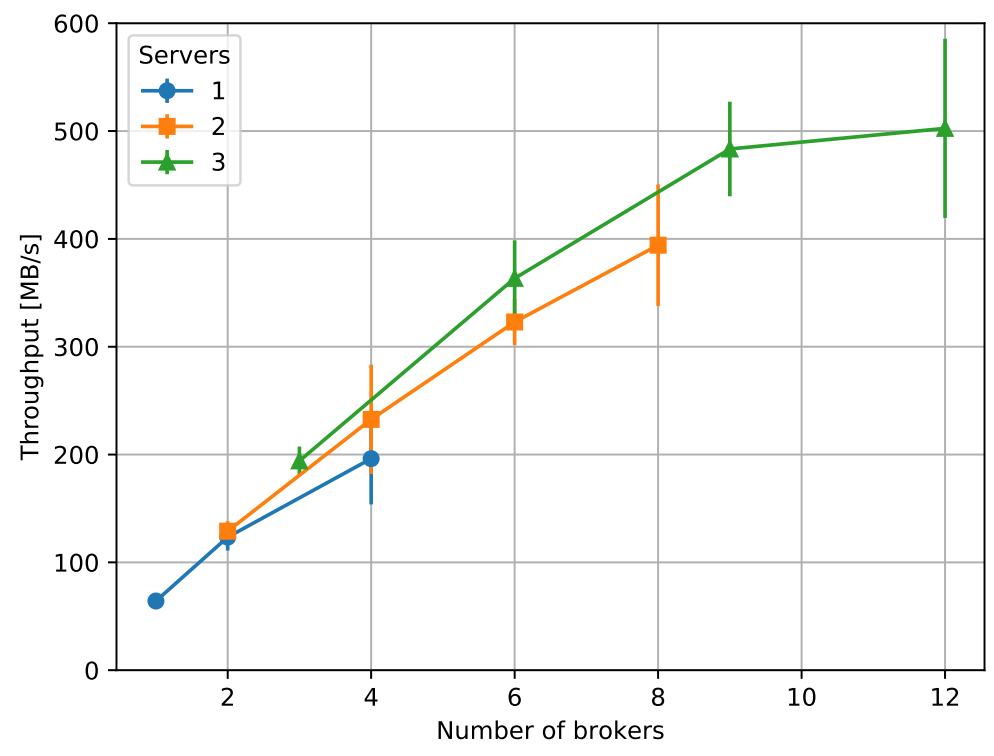

Figure 9. Scalability measurements: throughput as a function of the number of Kafka brokers with an event producer and a file writer, using one partition per broker.

\subsection{Deployments at operating facilities}

The data aggregation and streaming system was deployed in December 2017 to the V20 beamline at the Helmholtz-Zentrum Berlin's BER II research reactor. That deployment comprises a Kafka broker, the EPICS to Kafka Forwarder and the NeXus File Writer, along with applications for obtaining system metrics. Running the software infrastructure there allowed debugging the software and its configuration. An ESS mini-chopper was installed in the beamline and the system was able to obtain its data via EPICS and write them to file.

Another current deployment of the system is running at the STFC's ISIS Neutron and Muon Source. Three instruments are using the EPICS to Kafka Forwarder and a Kafka cluster. The system architecture differs from the ESS architecture in that the detector data do not come from EFUs, but from data acquisition electronics through the Instrument Control Program (ICP), together with metadata; moreover, the ICP is responsible for writing NeXus files. The EPICS forwarder is used to send beamline metadata to Kafka, while the Mantid Live Listener consumes live data from the cluster. Grafana is also being used for metrics visualisation. In two of the instruments, the system is being run in parallel with the current facility system, but in one of them, which is a new instrument, the Kafkaenabled ICP is the main control program. The ISIS Kafka cluster runs on three servers with Xeon R5$26202.10 \mathrm{GHz} 8$-core processors, $32 \mathrm{~GB}$ of RAM and three 1.2 TB disks in RAID 0 (for each server).

\section{Conclusion and future work}

The ESS will use a system architecture based on data aggregation and streaming for acquisition of neutron data in event mode and EPICS metadata, all of which will be timestamped at their source 
using the ESS timing system. An Apache Kafka cluster connects the applications producing and consuming these data, including Event Formation Units, Fast Sample Environment devices, EPICS Input/Output Controllers using the EPICS to Kafka Forwarder, Mantid and the NeXus File Writer. The solution can be tuned for balancing the throughput, latency and storage concerns arising from requirements, and also provides scalability and availability through partitioning and replication.

The Google FlatBuffers library is used for data serialisation and, with the schema definitions in a common code repository under source control, decouples the producer and consumer applications. This decoupling means parts of the system can be substituted by alternative components that use the appropriate schemas, making the system more generic and also usable at other neutron facilities.

Integration and performance tests have been run and demonstrate the viability of the solution for the ESS, showing the desired functionality and scalability behaviour. Deployments to operating neutron facilities, like the Helmholtz-Zentrum Berlin's BER II research reactor and the ISIS Neutron and Muon Source, corroborate this.

In the future the integration of the forwarder and file writer applications with the Experiment Control Program will be improved and the whole system tested for robustness in a number of failure scenarios. End-to-end tests will be performed at the integration laboratory at the ESS, using real beamline equipment and detector readout systems, as well as at the V20 beamline at the HZB.

\section{Acknowledgments}

This work is partially funded by the European Union Framework Programme for Research and Innovation Horizon 2020, under grant agreement 676548.

\section{References}

[1] S. Peggs et al., ESS Technical Design Report, ESS-doc-274 [https://europeanspallationsource.se/ sites/default/files/downloads/2017/09/TDR_online_ver_all.pdf].

[2] Experimental Physics and Industrial Control System website, https://epics.anl.gov.

[3] Apache Kafka website, http://kafka.apache.org/.

[4] M. Könnecke, F.A. Akeroyd, H.J. Bernstein, A.S. Brewster, S.I. Campbell, B. Clausen et al., The NeXus data format, J. Appl. Crystallogr. 48 (2015) 301.

[5] M.J. Christensen et al., Software-based data acquisition and processing for neutron detectors at European Spallation Source - early experience from four detector designs, submitted to JINST [arXiv: 1807.03980$]$.

[6] Daquiri, https://doi.org/10.5281/zenodo.1298511.

[7] Streaming Data Types, https://doi.org/10.5281/zenodo.1298378.

[8] J. Cereijo García, T. Korhonen and J.H. Lee, Timing system at ESS, in Proceedings of the $16^{\text {th }}$ International Conference on Accelerator and Large Experimental Control Systems, Barcelona, Spain, 2017, pp. 618-620.

[9] A. Mukai, F. Akeroyd, M. Brambilla, M. Christensen, M. Clarke, M. Jones et al., Status of the Development of the Experiment Data Acquisition Pipeline for the European Spallation Source, in Proceedings of the $16^{\text {th }}$ International Conference on Accelerator and Large Experimental Control Systems, Barcelona, Spain, 2017, pp. 835-839. 
[10] O. Arnold et al., Mantid - Data analysis and visualization package for neutron scattering and $\mu S R$ experiments, Nucl. Instrum. Meth. A 764 (2014) 156 [arXiv: 1407.5860].

[11] A. Mukai and T. Richter, Design report data aggregator software, BrightnESS Deliverable Rep. 5.1. [https://doi.org/10.17199/BRIGHTNESS.D5.1].

[12] FlatBuffers website, http://google.github.io/flatbuffers/.

[13] Forward EPICS to Kafka, https://doi.org/10.5281/zenodo.1298374.

[14] EPICS V4 website, http://epics-pvdata.sourceforge.net/index.html.

[15] librdkafka code repository, https://github.com/edenhill/librdkafka.

[16] The HDF Group, Hierarchical Data Format, version 5, http://www.hdfgroup.org/HDF5/, 1997-2018.

[17] Kafka to NeXus, https://doi.org/10.5281/zenodo.1298376.

[18] NeXus Format, Base Class Definitions, http://download.nexusformat.org/doc/html/classes/base_classes/index.html, 1996-2018.

[19] J. Stahn and A. Glavic, Focusing neutron reflectometry: Implementation and experience on the TOF-reflectometer Amor, Nucl. Instrum. Meth. A 821 (2016) 44.

[20] MPI Forum website, https://www.mpi-forum.org.

[21] D. Werder, F. Akeroyd, M. Brambilla, M. Clarke, M. Jones, M. Koennecke et al., Epics Data Streaming and HDF File Writing for ESS Benchmarked Using the Virtual AMOR Instrument, Proceedings of the $16^{\text {th }}$ International Conference on Accelerator and Large Experimental Control Systems, Barcelona, Spain, 2017, pp. 1815-1819. 